\title{
ENERGETIC GALERKIN BOUNDARY ELEMENT METHOD FOR 2D ELASTODYNAMICS: INTEGRAL OPERATORS WITH WEAK AND STRONG SINGULARITIES
}

\author{
GIULIA DI CREDICO, ALESSANDRA AIMI \& CHIARA GUARDASONI \\ Department of Mathematical, Physical and Computer Sciences, University of Parma, Italy
}

\begin{abstract}
In this paper, we consider some elastodynamics problems in 2D unbounded domains, with soft scattering conditions at the boundary, and their solution by the Boundary Element Method (BEM). The displacement identifying the elastic wave propagation is represented by both direct and indirect boundary integral formulations, which depend on the traction or on the jump of the traction at the boundary of the propagation domain, respectively. We study the characteristic singularities of the single layer and the double layer integral operators, which are involved in the considered energetic weak forms. Some algorithmic considerations about the parallel implementation of the energetic BEM and the quadrature techniques applied to overcome the issues due to the weak and the strong singularities of the integration kernels are proposed. Numerical simulations follow, showing a comparison between the external displacements obtained by the indirect and the direct formulations.
\end{abstract}

Keywords: elastodynamics, energetic BEM, single layer operator, double layer operator, weakly singular kernel, strongly singular kernel.

\section{INTRODUCTION}

The study of elastodynamics has several applications, including mechanical and civil engineering problems, seismic risk assessment and geological soil analyses. Often these problems are approached by domain methods, however this is not an optimal choice if the propagation happens throughout an external and unbounded domain, since artificial boundary conditions need to be imposed, potentially causing spurious reflections. The Boundary Element Method (BEM), instead, is in this context an efficient numerical technique, because both outer and interior propagations can be incorporated in the approximate solution of an integral equation, defined on the boundary of the diffusion domain. In this paper, we implement an energetic version of the BEM, introduced and analyzed for elastodynamics in the pioneering work by Bécache and Ha-Duong [1], in order to overcome the instabilities arising from the application of the standard BEMs [2] and with the aim of extending the optimal results for soft scattering problems reported by some authors of this work in [3].

\section{MODEL PROBLEM}

We study the propagation of an elastic vectorial wave in a two-dimensional domain $\Omega_{e} \subset$ $\mathbb{R}^{2}$, considering both the following situations: $\Omega_{e}$ external to a bounded and simply connected domain $\Omega_{i}$ with Lipschitz boundary $\Gamma=\partial \Omega_{i}$, namely $\Omega_{e}=\mathbb{R}^{2} \backslash \bar{\Omega}_{i}$, or $\Omega_{e}$ external to an open arc $\Gamma$, namely $\Omega_{e}=\mathbb{R}^{2} \backslash \Gamma$. Under the assumption of null external body forces, the displacement field $\mathbf{u}(\mathbf{x}, t)=\left(u_{1}, u_{2}\right)^{\top}(\mathbf{x}, t), \mathbf{x}=\left(x_{1}, x_{2}\right)^{\top} \in \Omega_{e}$, satisfies the Navier equation written in terms of displacement components [4]:

$$
\sum_{h, k, l=1}^{2} \frac{\partial}{\partial x_{h}}\left(C_{i h}^{k l} \frac{\partial u_{k}}{\partial x_{l}}(\mathbf{x}, t)\right)-\varrho \ddot{u}_{i}(\mathbf{x}, t)=0, \mathbf{x} \in \Omega_{e}, t \in(0, T], i=1,2
$$


where $\varrho$ is the mass density and the upper dots indicate derivatives with respect to time. The Hooke tensor $C_{i h}^{k l}=\lambda \delta_{i h} \delta_{k l}+\mu\left(\delta_{i k} \delta_{h l}+\delta_{i l} \delta_{h k}\right)$ depends on the Kronecker Delta $\delta_{i j}$ and on the positive Lamé parameters $\lambda$ and $\mu$, incorporating physical information about the diffusion domain. $T$ is the final time instant of analysis. We further introduce the traction field $\mathbf{p}(\mathbf{x}, t)=\left(p_{1}, p_{2}\right)^{\top}(\mathbf{x}, t)$ defined on the obstacle $\Gamma$ :

$$
p_{i}(\mathbf{x}, t)=\sum_{h, k, l=1}^{2} C_{i h}^{k l} \frac{\partial u_{k}}{\partial x_{l}}(\mathbf{x}, t) n_{\mathbf{x} h}, \quad \mathbf{x} \in \Gamma, t \in(0, T], i=1,2,
$$

where $\mathbf{n}_{\mathbf{x}}=\left(n_{\mathbf{x} 1}, n_{\mathbf{x} 2}\right)^{\top}$ is the unit outward normal vector to $\Gamma$ (if $\Gamma$ is an open arc and if $\Gamma^{-}$ and $\Gamma^{+}$denote respectively the lower and the upper sides of the obstacle, then $\mathbf{n}_{\mathbf{x}}$ is oriented from $\Gamma^{-}$to $\Gamma^{+}$). Eqn (1) is complemented by initial vanishing conditions and by Dirichlet boundary conditions, modelling a soft scattering by the obstacle, i.e.:

$$
\begin{array}{lr}
\mathbf{u}(\mathbf{x}, 0)=\dot{\mathbf{u}}(\mathbf{x}, 0)=0, & \mathbf{x} \in \Omega_{e}, \\
\mathbf{u}(\mathbf{x}, t)=\mathbf{g}(\mathbf{x}, t), \quad(\mathbf{x}, t) \in \Sigma:=\Gamma \times(0, T] .
\end{array}
$$

3 REPRESENTATION FORMULA AND BOUNDARY INTEGRAL EQUATIONS To describe the unknown $\mathbf{u}$ in $\Omega_{e} \times(0, T]$, we consider both direct and indirect integral representation equations:

$$
\begin{gathered}
\mathbf{u}(\mathbf{x}, t)=\mathcal{K} \mathbf{u}(\mathbf{x}, t)-\mathcal{V} \mathbf{p}(\mathbf{x}, t), \\
\mathbf{u}(\mathbf{x}, t)=\mathcal{V} \boldsymbol{\phi}(\mathbf{x}, t)
\end{gathered}
$$

The direct eqn (4) depends on the behaviour of the traction field $\mathbf{p}$ on $\Gamma$, while in the indirect eqn (5) the unknown density vector $\boldsymbol{\phi}=\left(\phi_{1}, \phi_{2}\right)^{\top}$ corresponds to the jump of $\mathbf{p}$ along the arc $\Gamma$. Setting a Dirichlet condition as in eqn (3), the external problem can be solved using, alternatively, eqns (4) or (5). The single layer operator $\mathcal{V}$ and the double layer operator $\mathcal{K}$ act on a generic field $\boldsymbol{\psi}$ defined on $\Sigma$ as follows:

$$
\begin{array}{r}
{[\mathcal{V} \Psi]_{i}(\mathbf{x}, t)=\sum_{j=1}^{2} \int_{0}^{t} \int_{\Gamma} G_{i j}(\mathbf{x}, \xi ; t, \tau) \psi_{j}(\xi, \tau) d \Gamma_{\xi} d \tau,} \\
{[\mathcal{K} \Psi]_{i}(\mathbf{x}, t)=\sum_{j=1}^{2} \int_{0}^{t} \int_{\Gamma} \sum_{h, k, l=1}^{2} C_{j h}^{k l} \frac{\partial G_{i j}}{\partial \xi_{l}}(\mathbf{x}, \xi ; t, \tau) \psi_{j}(\xi, \tau) n_{h \xi} d \Gamma_{\xi} d \tau,} \\
(\mathbf{x}, t) \in \Omega_{e} \times(0, T], i=1,2 .
\end{array}
$$

The second order tensor $G_{i j}$ in eqn (6) is the Green tensor, that is, the fundamental solution of the considered differential problem:

$$
\begin{aligned}
G_{i j}(\mathbf{x}, \xi ; t, \tau):= & \frac{H\left[c_{P}(t-\tau)-r\right]}{2 \pi \varrho c_{P}}\left\{\frac{r_{i} r_{j}}{r^{4}} \frac{2 c_{P}^{2}(t-\tau)^{2}-r^{2}}{\sqrt{c_{P}^{2}(t-\tau)^{2}-r^{2}}}-\frac{\delta_{i j}}{r^{2}} \sqrt{c_{P}^{2}(t-\tau)^{2}-r^{2}}\right\} \\
& -\frac{H\left[c_{S}(t-\tau)-r\right]}{2 \pi \varrho c_{S}}\left\{\frac{r_{i} r_{j}}{r^{4}} \frac{2 c_{S}^{2}(t-\tau)^{2}-r^{2}}{\sqrt{c_{S}^{2}(t-\tau)^{2}-r^{2}}}-\frac{\delta_{i j}}{r^{2}} \frac{c_{S}^{2}(t-\tau)^{2}}{\sqrt{c_{S}^{2}(t-\tau)^{2}-r^{2}}}\right\},
\end{aligned}
$$


where $H[\cdot]$ is the Heaviside function and where $c_{P}=\sqrt{(\lambda+2 \mu) / \varrho}>0$ and $c_{S}=\sqrt{\mu / \varrho}>0$ are the fundamental velocities of the irrotational and rotational displacements in which the vectorial solution of eqn (1) can be decomposed. The Green tensor $G_{i j}$ is symmetric w.r.t the space variables since it depends on them only through the vector $\mathbf{r}=\left(r_{1}, r_{2}\right)^{\top}=\mathbf{x}-\boldsymbol{\xi}=$ $\left(x_{1}-\xi_{1}, x_{2}-\xi_{2}\right)^{\top}$ and on its norm $r=\|\mathbf{r}\|_{2}$, namely the distance between the field $\mathbf{x}$ and the source point $\xi$. Considering a point $\mathbf{x} \in \Gamma$, from the representation eqns (4) and (5), we obtain the following Boundary Integral Equations (BIEs):

$$
\begin{gathered}
\mathcal{V} \mathbf{p}(\mathbf{x}, t)=\left(\mathcal{K}-\frac{1}{2} \mathcal{J}\right) \mathbf{g}(\mathbf{x}, t), \quad(\mathbf{x}, t) \in \Sigma, \\
\mathcal{V} \boldsymbol{\phi}(\mathbf{x}, t)=\mathbf{g}(\mathbf{x}, t), \quad(\mathbf{x}, t) \in \Sigma,
\end{gathered}
$$

where $\mathcal{J}$ is the identity operator and $\mathbf{g}=\left(g_{1}, g_{2}\right)^{\top}$ is the boundary datum assigned in eqn (3).

\section{ENERGETIC WEAK FORMULATION AND SPACE-TIME DISCRETIZATION}

In this work we reformulate the integral eqns (8) and (9) in the so called energetic weak form: this approach, already analyzed for scalar wave propagation problems [5] and introduced for soft scattering elastodynamic problems in [3], allows one to overcome the instabilities arising from the standard weak form of the BIEs. Therefore, having set $W=$ $\left(L^{2}\left([0, T] ; H^{-1 / 2}(\Gamma)\right)\right)^{2}$, the weak problems we intend to solve are as follows:

find $\boldsymbol{\varphi} \in W$ such that

$$
\langle\mathcal{V} \boldsymbol{\varphi}, \dot{\psi}\rangle_{L^{2}(\Sigma)}=\langle\overline{\mathbf{g}}, \dot{\boldsymbol{\psi}}\rangle_{L^{2}(\Sigma)}, \forall \boldsymbol{\psi} \in W,
$$

where $\boldsymbol{\varphi}$ coincides with $\mathbf{p}$ or $\boldsymbol{\phi}$ and $\overline{\mathbf{g}}$ coincides with $(\mathcal{K}-1 / 2 \mathcal{J}) \mathbf{g}$ or $\mathbf{g}$ in case we are solving eqn (8) or (9) respectively.

To solve the weak problem (10) in a discretized form, we first consider a uniform decomposition of the time interval [0,T], with time step $\Delta t=T / N_{\Delta t}, N_{\Delta \mathrm{t}} \in \mathbb{N}^{+}$and setting $N_{\Delta t}+1$ time-knots $t_{n}=n \Delta t, n=0, \ldots, N_{\Delta t}$. Having defined $\mathcal{P}_{d}$ as the space of algebraic polynomials of degree $d$, we define the corresponding space of piecewise polynomial functions of degree $d_{t}$ in time

$$
\mathcal{T}_{\Delta t, d_{t}}=\left\{\sigma:\left.\sigma\right|_{\left[t_{k}, t_{k+1}\right]} \in \mathcal{P}_{d_{t}} \forall_{k=0}^{N \Delta t-1}, \sigma \in C^{0}([0, T]) \text { and } \sigma(0)=0 \text { if } d_{t} \geq 1\right\}
$$

For the space discretization we introduce a boundary mesh constituted by a set of straight consecutive elements $\mathcal{E}=\left\{e_{1}, \ldots, e_{M}\right\}$ with length $2 l_{i}$ : $=\operatorname{length}\left(e_{i}\right) \leq \Delta x, e_{i} \cap e_{j}=\varnothing$ if $\neq$ $j$. For a piecewise linear arc, the union of the mesh elements is equivalent to $\Gamma$, or it represents a suitable approximation of the boundary in other cases. We have to choose functions in $H^{-1 / 2}(\Gamma)$ for the spatial approximation, therefore we consider the space of piecewise polynomial functions

$$
X_{\Delta x, d_{x}}=\left\{w \in L^{2}(\Gamma):\left.w\right|_{e_{i}} \in \mathcal{P}_{d_{x}}, e_{i} \in \mathcal{E}\right\}
$$

The Galerkin approximation of the energetic weak forms (10) in these spaces, having set $W_{\Delta x, \Delta t}=\left(X_{\Delta x, d_{x}} \otimes \mathcal{T}_{\Delta t, d_{t}}\right)^{2}$, reads: 
Find $\boldsymbol{\varphi}_{\Delta x, \Delta t} \in W_{\Delta x, \Delta t}$ such that

$$
\left\langle\mathcal{V} \boldsymbol{\varphi}_{\Delta x, \Delta t}, \dot{\Psi}_{\Delta x, \Delta t}\right\rangle_{L^{2}(\Sigma)}=\left\langle\overline{\mathbf{g}}, \dot{\Psi}_{\Delta x, \Delta t}\right\rangle_{L^{2}(\Sigma)}, \forall \boldsymbol{\Psi}_{\Delta x, \Delta t} \in W_{\Delta x, \Delta t}
$$

We introduce the set $\left\{w_{m}(\mathbf{x})\right\}_{m=1}^{M_{\Delta x}}$, containing the basis functions of the space $X_{\Delta x, d_{x}}$, which are piecewise polynomials depending on the Lagrangian polynomials on each element $e_{i}$. For the time discretization we choose piecewise constant basis functions $\left(d_{t}=0\right)$ :

$$
\chi_{n}(t)=H\left[t-t_{n}\right]-H\left[t-t_{n+1}\right], \quad n=0, \ldots, N_{\Delta t}-1 .
$$

Hence, the components of the discrete function $\boldsymbol{\varphi}_{\Delta x, \Delta t}$ can be expressed in space and time as

$$
\varphi_{\Delta x, \Delta t}^{i}(\mathbf{x}, t)=\sum_{n=0}^{N_{\Delta t}-1} \sum_{m=1}^{M_{\Delta x}} \alpha_{n m}^{i} w_{m}(\mathbf{x}) \chi_{n}(t), \quad i=1,2 .
$$

Replacing the test functions with the space-time basis functions defined above, the Galerkin eqn (11) leads to the linear system $E \boldsymbol{\alpha}=\boldsymbol{\beta}$, with the following block structure

$$
\left(\begin{array}{cccc}
E^{(0)} & 0 & \cdots & 0 \\
E^{(1)} & E^{(0)} & \cdots & 0 \\
\vdots & \vdots & \ddots & \vdots \\
E^{\left(N_{\Delta t}-1\right)} & E^{\left(N_{\Delta t}-2\right)} & \cdots & E^{(0)}
\end{array}\right)\left(\begin{array}{c}
\boldsymbol{\alpha}_{0} \\
\boldsymbol{\alpha}_{1} \\
\vdots \\
\boldsymbol{\alpha}_{N_{\Delta t}-1}
\end{array}\right)=\left(\begin{array}{c}
\boldsymbol{\beta}_{0} \\
\boldsymbol{\beta}_{1} \\
\vdots \\
\boldsymbol{\beta}_{N_{\Delta t}-1}
\end{array}\right)
$$

where for all $l=0, \ldots, N_{\Delta t}-1$ the $l$-th block and the $l$-th entry of the solution vector are organized as

$$
E^{(l)}=\left(\begin{array}{ll}
E_{11}^{(l)} & E_{12}^{(l)} \\
E_{21}^{(l)} & E_{22}^{(l)}
\end{array}\right), \quad \boldsymbol{\alpha}^{(l)}=\left(\begin{array}{llllll}
\alpha_{l 1}^{1} & \cdots & \alpha_{l M_{\Delta x}}^{1} & \alpha_{l 1}^{2} & \cdots & \alpha_{l M_{\Delta x}}^{2}
\end{array}\right) .
$$

The structure of the right-hand side $\boldsymbol{\beta}$ is analogous to that of the solution vector.

Solving eqn (12) by blockwise forward substitution leads to a marching-on-in-time (MOT) time stepping scheme. The double analytical integration in the time variables, leads to the matrix entry

$$
\left(E_{i j}^{(l)}\right)_{\widetilde{m}, m}=-\sum_{\zeta, \varsigma=0}^{1} \frac{(-1)^{\zeta+\varsigma}}{2 \pi \varrho} \int_{\Gamma} \int_{\Gamma} w_{\widetilde{m}}(\mathbf{x}) w_{m}(\xi) v_{i j}\left(r ; \Delta_{\tilde{n}+\zeta, n+\varsigma}\right) d \Gamma_{\xi} d \Gamma_{\mathbf{x}}
$$

for all $i, j=1,2, m, \widetilde{m}=1, \ldots, M_{\Delta x}$ and $n, \tilde{n}=0, \ldots, N_{\Delta t}-1$, where the positive time difference $t_{\tilde{n}+\zeta}-t_{n+\varsigma}=\Delta_{\tilde{n}+\zeta, n+\varsigma}$, with $\tilde{n}-n=l$, is set. For a generic $\Delta>0$, the kernel $v_{i j}$ is defined as

$$
\begin{aligned}
v_{i j}(r ; \Delta):=\left(\frac{r_{i} r_{j}}{r^{4}}-\right. & \left.\frac{\delta_{i j}}{2 r^{2}}\right)\left[\frac{H\left[c_{P} \Delta-r\right]}{c_{P}} \Delta h_{P, 1}(r ; \Delta)-\frac{H\left[c_{S} \Delta-r\right]}{c_{S}} \Delta h_{S, 1}(r ; \Delta)\right] \\
& +\frac{\delta_{i j}}{2}\left[\frac{H\left[c_{P} \Delta-r\right]}{c_{P}^{2}} h_{P, 2}(r ; \Delta)+\frac{H\left[c_{S} \Delta-r\right]}{c_{S}^{2}} h_{S, 2}(r ; \Delta)\right],
\end{aligned}
$$

with the specific kernel functions given by 
$h_{\gamma, 1}(r ; \Delta):=\sqrt{c_{\gamma}^{2} \Delta^{2}-r^{2}}, \quad h_{\gamma, 2}(r ; \Delta):=\log \left(\sqrt{c_{\gamma}^{2} \Delta^{2}-r^{2}}+c_{\gamma} \Delta\right)-\log (r), \quad \gamma=S, P$.

If $0 \leq r \leq c_{S} \Delta<c_{P} \Delta$ all the arguments of the Heaviside functions in eqn (14) are positive and the kernel $v_{i j}$ can be expressed in a reduced form with space singularity of kind $\mathcal{O}(\log (r))$ for $r \rightarrow 0$. This behaviour is well-studied for boundary integral operators related to $2 \mathrm{D}$ elliptic problems. If we choose to solve the discretized weak form arising from eqn (9), the right hand side $\boldsymbol{\beta}$ has elements made by the $L^{2}(\Sigma)$ product of the Dirichlet datum $\mathbf{g}$ by the time derivative of the basic functions in space and time. Otherwise, starting from eqn (8), the product $\langle\mathcal{K} \mathbf{g}, \dot{\Psi}\rangle_{L^{2}(\Sigma)}$ is evaluated by an approximation of the two components of the boundary datum with a linear combination of space-time basis functions:

$$
g_{i}(\mathbf{x}, t) \approx \hat{g}_{i}(\mathbf{x}, t):=\sum_{n=0}^{N_{\Delta t}-1} \sum_{m=1}^{M_{\Delta x}} \hat{g}_{n m}^{i} w_{m}(\mathbf{x}) v_{n}(t), \quad i=1,2,
$$

where

$$
v_{n}(t)=H\left[t-t_{n}\right] \frac{t-t_{n}}{\Delta t}-H\left[t-t_{n+1}\right] \frac{t-t_{n+1}}{\Delta t}, \quad n=0, \ldots, N_{\Delta t}-1
$$

are basis functions of $\mathcal{T}_{\Delta t, 1}$. To have a correct interpolation of the boundary datum, the condition $\hat{g}_{i}\left(\mathbf{x}_{m}, t_{k}\right)=g_{i}\left(\mathbf{x}_{m}, t_{k}\right)$ is imposed: the spatial nodes $\mathbf{x}_{m}$ are the middle points of the mesh elements $e_{m}$ if $w_{m}$ are piecewise constant, otherwise are the amount of points determined by the interpolation nodes of the Lagrangian polynomials defining spatial shape functions of higher degree. With this substitution, the evaluation of $\langle\mathcal{K} \mathbf{g}, \dot{\boldsymbol{\psi}}\rangle_{L^{2}(\Sigma)}$ becomes the computation of the matrix-vector product $\hat{E} \hat{\mathbf{g}}$, with $\widehat{E}$ having similar Toeplitz structure as $E$ and $\hat{\mathbf{g}}$ column vector with elements $\hat{g}_{n m}^{i}$. Also for the double layer operator, we can perform an analytical time integration, leading to the matrix entry

$$
\left(\hat{E}_{i j}^{(l)}\right)_{\widetilde{m}, m}=\sum_{\zeta, \varsigma=0}^{1} \frac{(-1)^{\zeta+\varsigma}}{\pi \varrho \Delta t} \int_{\Gamma} \int_{\Gamma} w_{\widetilde{m}}(\mathbf{x}) w_{m}(\xi) \hat{v}_{i j}\left(r ; \Delta_{\tilde{n}+\zeta, n+\varsigma}\right) d \Gamma_{\xi} d \Gamma_{\mathbf{x}}
$$

for all $i, j=1,2, m, \widetilde{m}=1, \ldots, M_{\Delta x}$ and $n, \tilde{n}=0, \ldots, N_{\Delta t}-1$. The integration kernel $\hat{v}_{i j}$, for a positive $\Delta$, is defined as follows

$$
\begin{gathered}
\hat{v}_{i j}(r ; \Delta):=\sum_{h, k, l=1}^{2} C_{j h}^{k l}\left(-\frac{A_{i k l}}{2 r} \frac{h_{S, 1}(r ; \Delta)}{c_{S}^{3}} H\left[c_{S} \Delta-r\right]\right. \\
+\frac{1}{6 r} B_{i k l}\left[\frac{h_{S, 1}(r ; \Delta)}{c_{S}^{3}} H\left[c_{S} \Delta-r\right]-\frac{h_{P, 1}(r ; \Delta)}{c_{P}^{3}} H\left[c_{P} \Delta-r\right]\right] \\
+\frac{\Delta^{2}}{6 r^{3}} D_{i k l}\left[\frac{h_{P, 1}(r ; \Delta)}{c_{P}} H\left[c_{P} \Delta-r\right]-\frac{h_{S, 1}(r ; \Delta)}{c_{S}} H\left[c_{S} \Delta-r\right]\right] n_{h \xi},
\end{gathered}
$$

with $A_{i k l}=\delta_{i k} r_{, l}, B_{i k l}=\delta_{i l} r_{, k}+\delta_{k l} r_{, i}+\delta_{i k} r_{, l}-r_{, i} r_{, k} r_{, l}, D_{i k l}=\delta_{i l} r_{, k}+\delta_{k l} r_{, i}+\delta_{i k} r_{, l}-$ $4 r_{, i} r_{, k} r_{, l}$ and $r_{, k}=r_{k} / r$ for $k=1,2$. If $0 \leq r \leq c_{S} \Delta<c_{P} \Delta$, the kernel $\hat{v}_{i j}$ reduces to a simplified form with strong singularity $\mathcal{O}(1 / r)$ for $r \rightarrow 0$. 


\section{PARALLEL COMPUTATION OF TOEPLITZ MATRICES}

The construction of the linear system (12) requires just the computation of the first column of blocks of $E$ and this is a great advantage in terms of computations saving and memory requirement. Moreover, the computation of the matrix blocks $E^{(l)}$ and of the right hand side $\boldsymbol{\beta}_{l}$ is embarrassingly parallel. For this reason, the entire method can be improved in programming phase with a parallel computing approach. The elapsed times decay reported in Fig. 1 refers to the construction of 400 time blocks of $E$ and $\boldsymbol{\beta}$, each depending on 320 spatial degrees of freedom. Increasing the number of threads used to compute every block of the linear system leads to a linear decline of the execution times.

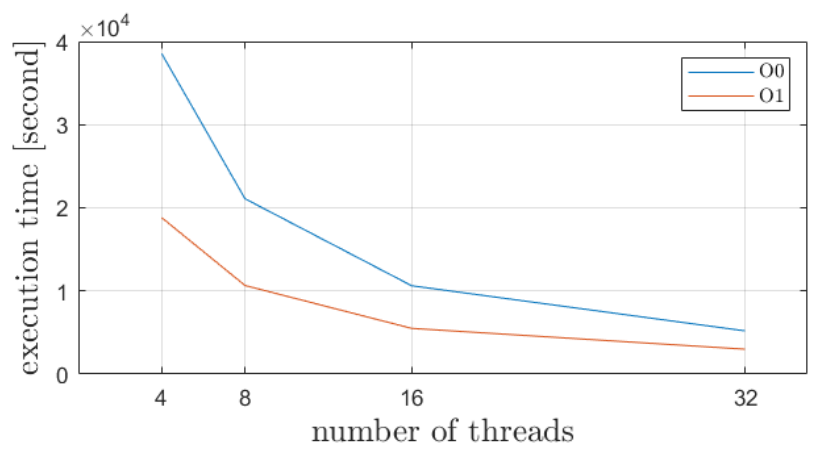

Figure 1: Scalability of the algorithm that computes the time blocks of the matrix and of the right hand side of the linear system $E \boldsymbol{\alpha}=\boldsymbol{\beta}$ (in eqn (11) $\overline{\mathbf{g}}=\mathbf{g}$ is set).

In particular the method is implemented by the parallelization of a Fortran code with open-mp library. The labels 00,01 in Fig. 1 refer to different optimization setup of the Intel compiler: the options $O 0$ is the default, the option $O 1$ maximizes speed by negligibly reducing the accuracy, leading to a faster parallel computation of the time block matrices. If the problem is solved with a direct approach, similar considerations can be repeated for the parallel computation of the double layer matrix $\hat{E}$.

\section{INTEGRATION TECHNIQUES}

Following the element by element technique, we calculate the double integral in eqn (13), for a positive time difference $\Delta$, as the sum of local contributions of type

$$
\int_{0}^{2 l_{\widetilde{m}}} \int_{0}^{2 l_{m}} w_{\widetilde{m}}^{\left(d_{x}\right)}(s) w_{m}^{\left(d_{x}\right)}(z) v_{i j}(r(s, z) ; \Delta) d z d s,
$$

obtained after the parametrization of the generic integration segments $e_{m}$ and $e_{\widetilde{m}}$ belonging to $\Gamma$ discretization (see Section 4):

$$
\mathbf{x} \rightarrow s \in\left[0,2 l_{m}\right], \quad \xi \rightarrow z \in\left[0,2 l_{\tilde{m}}\right],
$$

and where $w_{\widetilde{m}}^{\left(d_{x}\right)}(s)$ and $w_{m}^{\left(d_{x}\right)}(z)$ are local Lagrangian basis functions in the space variable defined over the elements $e_{\widetilde{m}}$ and $e_{m}$. The double integral in eqn (15) can be written in the same way by substituting $v_{i j}$ with $\hat{v}_{i j}$. An important integration issue is the dependence of both kernels $v_{i j}$ and $\hat{v}_{i j}$ on the Heaviside functions $H\left[c_{\gamma} \Delta-r\right]$, which define the wavefronts 
with peculiar velocities $c_{S}$ and $c_{P}$. To avoid the integration of these discontinuous functions, the local rectangular domain of integration $\left[0,2 l_{\widetilde{m}}\right] \times\left[0,2 l_{m}\right]$ in eqn $(16)$ is further divided into regions depending on the splitting points determined by the equation $r=c_{\gamma} \Delta, \gamma=S, P$, where the distance $r$ can assume different expressions:

$e_{\widetilde{m}} \equiv e_{m} \Rightarrow r=|s-z|$,

$e_{\tilde{m}}, e_{m}$ consecutive and aligned $(m<\tilde{m}) \Rightarrow r=s+2 l_{m}-z$,

$e_{\tilde{m}}, e_{m}$ consecutive and not aligned with angle $\theta(m<\widetilde{m})$

$$
\Rightarrow r^{2}=s^{2}+\left(2 l_{m}-z\right)^{2}-2 \cos (\theta) s\left(2 l_{m}-z\right) \text {. }
$$

An example of integration domains depending on the mutual position of the mesh elements is in Fig. 2, where in particular the sets $E_{S} \cap\left[0,2 l_{\tilde{m}}\right] \times\left[0,2 l_{m}\right]$ and $E_{S, P} \cap\left[0,2 l_{\tilde{m}}\right] \times\left[0,2 l_{m}\right]$, with

$$
E_{S}=\left\{r(s, z)<c_{S} \Delta\right\}, \quad E_{S, P}=\left\{c_{S} \Delta<r(s, z)<c_{P} \Delta\right\},
$$

are highlighted.

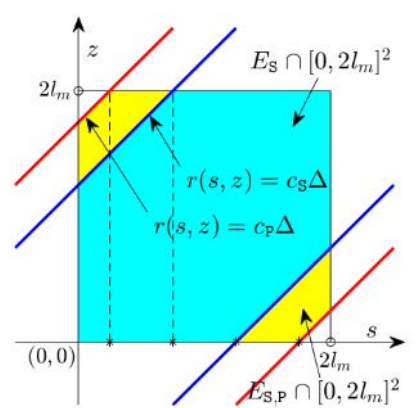

(a)

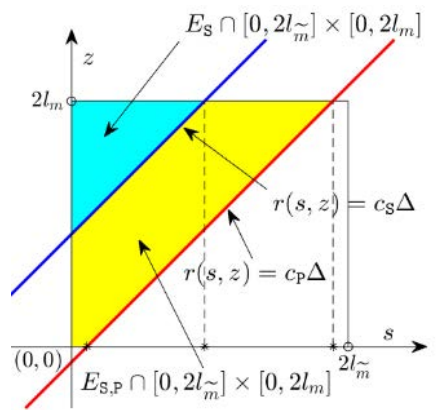

(b)

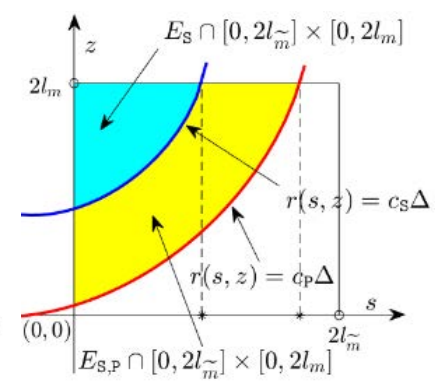

(c)

Figure 2: Example of integration domains in case of coincident elements (a), consecutive aligned elements (b) and consecutive not aligned elements (c).

Then, we calculate eqn (16) summing the contribution over each region, where $s$ belongs to a specific interval $[a, b] \subset\left[0,2 l_{\tilde{m}}\right]$, and the inner interval $[A(s), B(s)]$ strictly depends on the outer variable. A complete overview of the shape and the related splitting of the integration domains is reported in a recently submitted work [6].

We focus now on the single layer kernel $v_{i j}$, observing that it depends on the function $\log \left(c_{\gamma} \Delta+\sqrt{c_{\gamma}^{2} \Delta^{2}-r^{2}}\right), \gamma=S, P$, which is not singular but has an unbounded derivative for $r=c_{\gamma} \Delta$. Therefore, the accurate integration of this part of the kernel would require a really large number of Gauss-Legendre quadrature nodes. To avoid high computational costs, the inner integration of this function is computed by combining the Gaussian rule with a regularization procedure, analyzed in [7], involving the use of the following change of integration variable $=\Phi(\hat{z}), \hat{z} \in[0,1]$, where

$$
\Phi(\hat{z})=\frac{(p+q-1) !}{(p-1) !(q-1) !} \int_{0}^{\hat{z}} u^{p-1}(1-u)^{q-1} d u
$$


helps to move the quadrature nodes towards the endpoints of the interval (where it is possible to have $r=c_{\gamma} \Delta$ ), in order to approximate better the behaviour of the square root. The position of the nodes is regulated by the value of the characteristic exponents $p, q \geq 1$ of the equation. The outer integration in the $s$ variable can be performed with a standard Gauss-Legendre rule.

Further integration problems arise from logarithmic singularity. In case of coincident elements, where $r=|s-z|$ is null for $s=z$, it is possible to compute the inner integration with the quadrature equations proposed in [8], of the type

$$
\int_{-1}^{1} \log \left(\left|s-z_{G}\right|\right) f\left(z_{G}\right) d z_{G} \approx \sum_{i=1}^{n} \omega_{i}(s) f\left(z_{G, k}\right) .
$$

The weights in eqn (18) depend both on the weights of the Gauss-Legendre equation and on the singular kernel, while the nodes $z_{G, k}$ are the roots of the Legendre polynomials of degree $n$. The recursive relation to calculate $\omega_{i}(s)$ may be found in [8]. The equation is interpolatory, namely it calculates the integral exactly if the function $f$ is a polynomial up to degree $n-1$, making the procedure suitable for our purposes since the kernel in eqn (16) is multiplied by the basic function $w_{m}^{\left(d_{x}\right)}$. Moreover, eqn (18) can be easily adapted to other mutual positions of the mesh elements.

Concerning the double layer kernel $\hat{v}_{i j}$, we have to deal with the strong singularity $\mathcal{O}(1 / r)$. Let us suppose also in this case that we have to compute an integral of type (16) for coincident elements: this means that we have to calculate contributions in local variables of the following type

$$
I=\int_{a}^{b} \int_{A(S)}^{B(S)} w_{\widetilde{m}}^{\left(d_{x}\right)}(s) w_{m}^{\left(d_{x}\right)}(z) \frac{\sqrt{c_{S}^{2} \Delta^{2}-(s-z)^{2}}}{s-z} d z d s,
$$

where in particular the kernel is strongly singular at all points $(s, z)$ in the integration domain where $z=s$. If we operate a first-order Taylor expansion of the numerator of the interior integrand function for $z=s$, integral (19) becomes

$$
\begin{aligned}
I= & -\int_{a}^{b} w_{\widetilde{m}}^{\left(d_{x}\right)}(s) \int_{A(s)}^{B(s)} \frac{w_{m}^{\left(d_{x}\right)}(z) \sqrt{c_{S}^{2} \Delta^{2}-(s-z)^{2}}-c_{S} \Delta w_{m}^{\left(d_{x}\right)}(s)}{z-s} d z d s \\
& -\int_{a}^{b} w_{\widetilde{m}}^{\left(d_{x}\right)}(s) c_{S} \Delta w_{m}^{\left(d_{x}\right)}(s) \int_{A(S)}^{B(S)} \frac{1}{z-s} d z d s=I_{1}+I_{2} .
\end{aligned}
$$

Integral $I_{1}$ in eqn (20) is no more singular but it requires the regularization procedure (17) if the argument of $\sqrt{c_{S}^{2} \Delta^{2}-(s-z)^{2}}$ vanishes at the endpoints of the integration domain. Regarding the second term $I_{2}$, the inner integral can be computed exactly:

$$
\int_{A(s)}^{B(S)} \frac{1}{z-s} d z=\log (B(s)-s)-\log (s-A(s)) .
$$

The outer integration of $I_{2}$ presents an issue if the argument of the log functions in eqn (21) is null in one of the extremes of the interval $[a, b]$ (or in both). In case of these log mild singularities, we can perform the outer numerical integration combining a Gaussian quadrature rule with eqn (17), in order to suitably accumulate quadrature nodes towards the endpoints of integration. 


\section{NUMERICAL RESULTS}

In this section we present two experiments which allow us to compare the results given by the discretization of the two formulations resumed in eqn (10). Concerning the parameters employed in all the following tests, we set peculiar velocities $c_{S}=1$ and $c_{P}=2$ and mass density $\varrho=1$. The basis functions $\left\{w_{m}(\mathbf{x})\right\}_{m=1}^{M_{\Delta x}}$ for the space discretization are set to be piecewise constant (degree $d_{x}=0$ ), meaning that the number $M_{\Delta x}$ of spatial degrees of freedom is equivalent to the number of elements $e_{m}$ of the mesh over $\Gamma$. We recall that in this section we denote by $\mathbf{p}$ and $\boldsymbol{\phi}$ the approximations given by the solution of the energetic problem (11) related to the direct or indirect formulation respectively.

\subsection{Experiment 1}

In this example we consider a flat crack $\Gamma=\left\{\left(x_{1}, 0\right) \in \mathbb{R}^{2} \mid-0.5 \leq x_{1} \leq 0.5\right\}$ and the Dirichlet condition $\mathbf{g}\left(x_{1}, t\right)$ on $\Sigma$ such that $g_{1} \equiv 0$ and $g_{2}\left(x_{1}, t\right)=f(t+0.45)$, where the temporal profile $f(t)$ is defined as follows:

$$
f(t)=\left\{\begin{array}{lc}
t-1 / 2, & t \in[0.5,0.6] \\
(-30 t+19) / 10, & t \in[0.6,0.7] \\
(40 t-30) / 10, & t \in[0.7,0.8] \\
(-30 t+26) / 10, & t \in[0.8,0.9] \\
t-1, & t \in[0.9,1] \\
0 & \text { otherwise }
\end{array}\right.
$$

We set the final instant $T=4$ and we impose on $\Gamma$ a uniform decomposition with step $\Delta x=$ 0.025 (40 segments) and a time step of size $\Delta t=0.0125$. The boundary datum models a uniform scattering by the obstacle with vertical direction. In Fig. 3(a) and 3(b) the solution arrays $\mathbf{p}$ and $\boldsymbol{\phi}$ of the energetic weak form (11), considering the indirect and the direct formulations respectively, are shown: in particular there is the time history of the horizontal and the vertical components of $\mathbf{p}$ and $\boldsymbol{\phi}$ at the point $(-1 / 4,0) \in \Gamma$. It is possible to observe that $\phi_{1}$ is trivial. This is due to the fact that the condition $\mathbf{g}$ is completely vertical and, on a flat horizontal obstacle, the Green tensor defined in eqn (7) has null components for $i \neq j$.
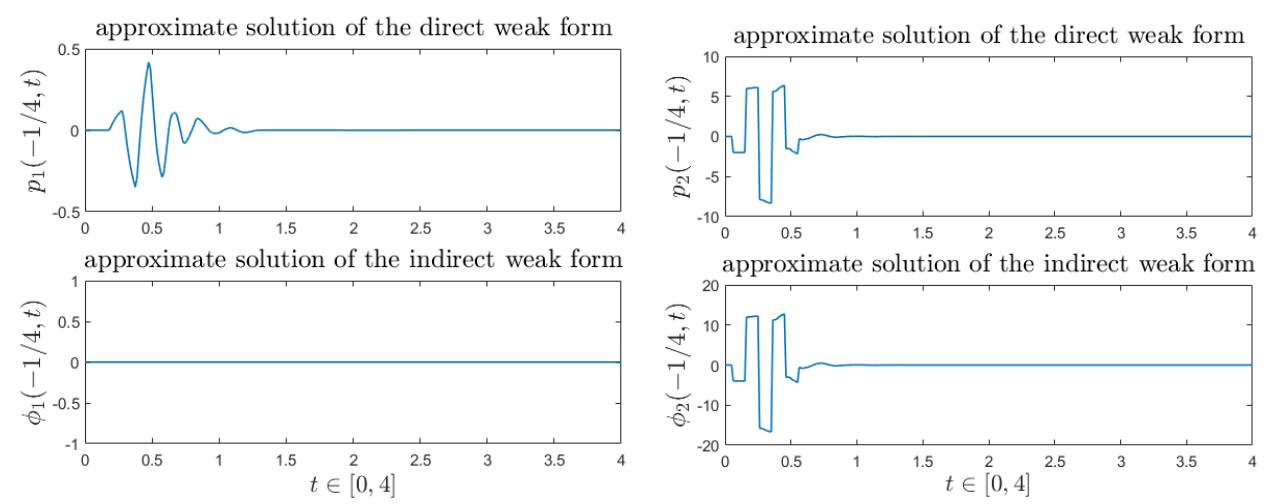

(a)

(b)

Figure 3: Horizontal and vertical components of the vector solutions $\mathbf{p}$ and $\boldsymbol{\phi}$ of the weak problem (11) calculated at point $(-1 / 4) \in \Gamma$ and during the time interval $[0,4]$. 
This leads to a reduction of the indirect BIE (9), which is decoupled in two scalar equations: $\mathcal{V}_{11} \phi_{1}=0$ and $\mathcal{V}_{22} \phi_{2}=g_{2}$. The right hand side of the direct weak form instead depends also on the double layer operator, meaning that the components of $\overline{\mathbf{g}}$ in eqn (11) are both active. Anyway, both approaches let us to calculate approximate results showing long time stability.

Solutions of the energetic weak formulations have then been employed in the respective integral representation eqns (4) and (5) to obtain the displacement $\mathbf{u}$ at the points of an axis orthogonal to $\Gamma$ in its middle point $(0,0)$. Snapshots of the vertical displacement at the time instants 1, 2 and 3 are shown in Fig. 4: in Fig. 4(a) the component $u_{2}$ is calculated replacing the approximate traction $\mathbf{p}$ in the direct eqn (4), while Fig. 4(b) have been obtained by the indirect eqn (5), leading to a comparable results for the external propagation.
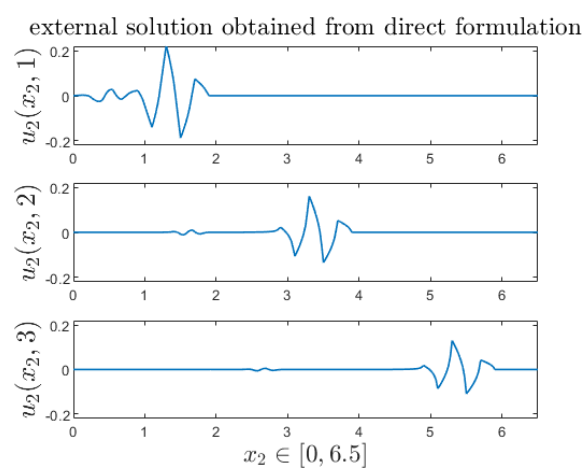

(a)
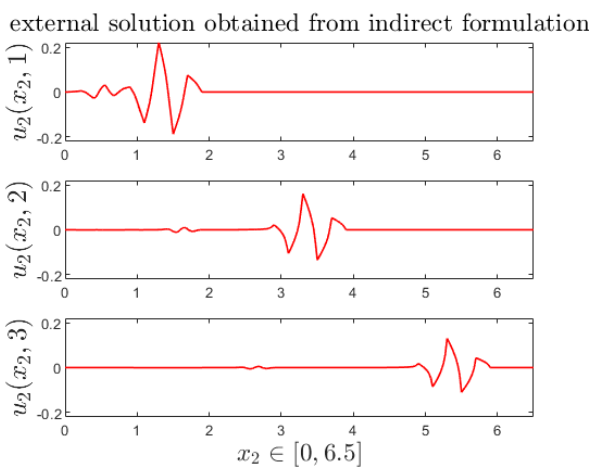

(b)

Figure 4: External displacement $\mathbf{u}$ calculated, for different time instants, along the vertical axes $\left(0, x_{2}\right), x_{2} \in[0,6.5]$. Solutions have been obtained by the discretization of the direct weak form (a) and the indirect weak form (b).

This results in a wave spreading with the peculiar velocity $c_{P}=2$, followed by a slower perturbation with speed $c_{S}=1$. This last one is a circular perturbation having origin in the endpoints of the open arc $\Gamma$, usually called in literature crack tips phenomena.

Lastly, a numerical comparison is reported in Table 1, where the numbers represent the difference in $L^{2}$ norm between the external displacements obtained by the two different formulations evaluated at different time instants. These errors are $\mathcal{O}\left(10^{-3}\right)$, as expected considering the magnitude of the imposed spatial and temporal discretization steps $\Delta x$ and $\Delta t$.

Table 1: $L^{2}$-error for $x_{2} \in[0,6.5]$ of the vertical components $u_{2}$ for some time instants.

$$
\begin{array}{|l|c|c|c|}
\hline\left\|u_{2, \text { direct }}(\cdot, t)-u_{2, \text { indirect }}(\cdot, t)\right\|_{L^{2}([0,6.5])} & t=1 & t=2 & t=3 \\
\cline { 2 - 4 } & 6.92 \cdot 10^{-3} & 8.15 \cdot 10^{-4} & 6.64 \cdot 10^{-4} \\
\hline
\end{array}
$$

\subsection{Experiment 2}

We now consider the circular arc $\Gamma=\left\{\left(x_{1}, x_{2}\right) \in \mathbb{R}^{2} \mid x_{1}^{2}+x_{2}^{2}=1\right\}$ and the Dirichlet condition $\mathbf{g}(\mathbf{x}, t)$ on $\Sigma$ such that $g_{1}(\mathbf{x}, t)=h(t) x_{1}$ and $g_{2}(\mathbf{x}, t)=h(t) x_{2}$, where the function $h(t)=0.2 \sin ^{2}(40 t) H[\pi / 40-t]$ is the temporal profile. 
We set the final instant $T=4$ and we impose on $\Gamma$ a uniform decomposition with step $\Delta x \simeq 0.02$ (320 segments) and we choose a time step of size $\Delta t=0.01$. With this problem geometry, the boundary datum models a uniform scattering by the obstacle with radial direction with respect to the centre of $\Gamma$. In Fig. 5 there is the time history of the vertical components of $\mathbf{p}$ and $\boldsymbol{\phi}$, obtained respectively by the discretized direct and indirect weak form (11), at the point $(0,1) \in \Gamma$. As we are solving an external problem, the traction $\mathbf{p}$ on $\Gamma$ presents just an initial perturbation of length $\pi / 40$, effect of the pulse generated by the Dirichlet boundary condition (see Fig. 5(a)). Differently, the density $\boldsymbol{\phi}$ in Fig. 5(b) presents a series of pulsations since it depends also on the interior wave reflections against $\Gamma$. In Fig. 6 , three snapshots of the vertical external displacement $\mathbf{u}$ are represented, obtained respectively by the discretized direct and indirect weak form (11) (Fig. 6(a) and 6(b)), along the vertical axes $\left(0, x_{2}\right), x_{2} \in[1,8]$. Externally we have the diffusion of a pure radial perturbation with the peculiar velocity $c_{P}=2$. There are no crack tips perturbations since the arc is closed.

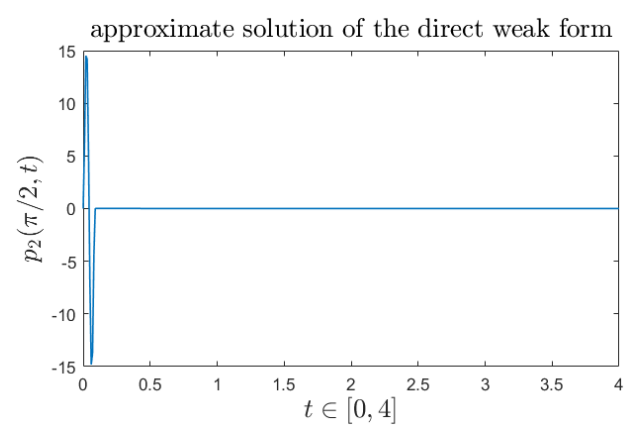

(a)

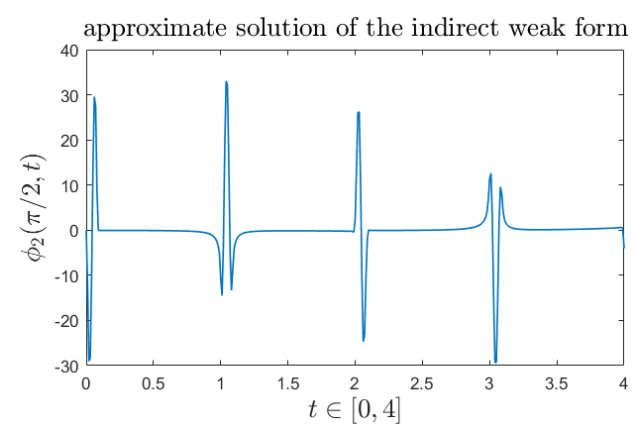

(b)

Figure 5: Vertical components of the vector solutions $\mathbf{p}$ and $\boldsymbol{\phi}$ of the weak problem (11) calculated at point $(0,1) \in \Gamma$ (corresponding to the clockwise angle $\pi / 2)$ and during the time interval $[0,4]$.

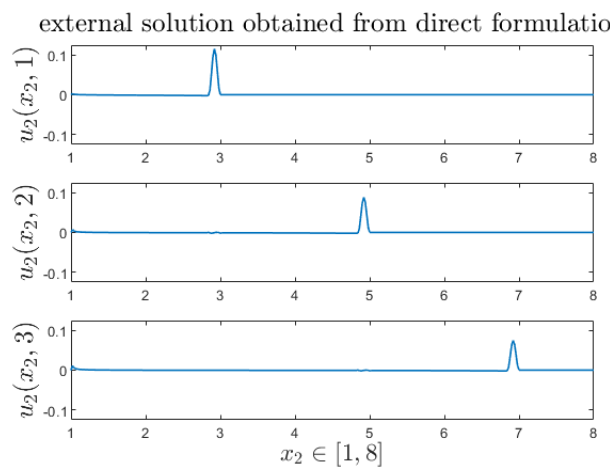

(a)
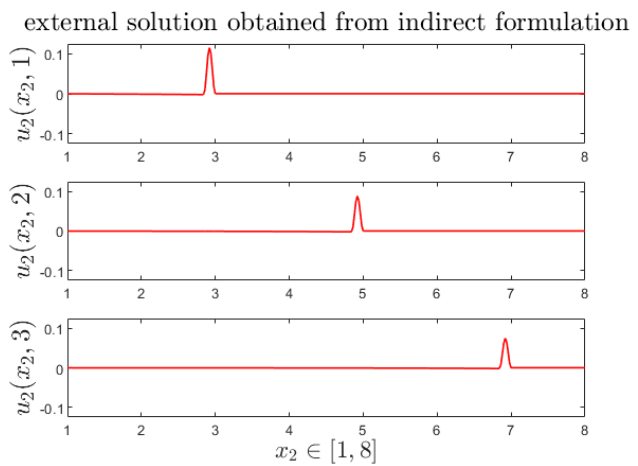

(b)

Figure 6: Vertical displacement $u_{2}$ calculated, for different time instants, along the vertical axes $\left(0, x_{2}\right), x_{2} \in[1,8]$. Solutions have been obtained by the discretization of the direct weak form (a) and the indirect weak form (b). 
Also for the circular obstacle, we have a numerical comparison in Table 2, where the difference in $L^{2}$-norm between the external displacements obtained by the two different formulations is reported. Also in this case the errors are consistent with the fixed discretization space-time steps.

Table 2: $L^{2}$-error for $x_{2} \in[1,8]$ of the vertical components $u_{2}$ for some time instants.

$$
\begin{array}{|l|c|c|c|}
\left\|u_{2, \text { direct }}(\cdot, t)-u_{2, \text { indirect }}(\cdot, t)\right\|_{L^{2}([1,8])} & t=1 & t=2 & t=3 \\
\cline { 2 - 4 } & 5.32 \cdot 10^{-4} & 1.36 \cdot 10^{-3} & 2.07 \cdot 10^{-3} \\
\hline
\end{array}
$$

\section{CONCLUSIONS}

The comparison between the energetic BEM approximations of representation eqns (4) and (5) for the elastodynamic displacement, with soft scattering conditions, is the focus of this research.

By energetic BEM, the numerical results shown in Section 7 (concerning wave propagation problems external to a straight crack and to a closed circular domain) are achieved directly in the space-time domain allowing a better comprehension of the behaviour at the boundary of the solutions of the related direct and indirect BIEs (8) and (9).

The long-time stability of the results is independent of the discretization parameters. As described in Section 6, the efficiency and the accuracy of the entire energetic method can be equivalently maintained in both formulations by using suitable integration routines to treat the spatial singularities involved in the single and the double layer integration kernels and by the study of a proper splitting of the integration domains, determined by the two wave fronts characteristic of the elastic propagation. The direct eqn (4), even if more costly, will allow us to also handle the extension to mixed boundary condition problems.

As shown, the linear systems outcome of the energetic BEM applied to elastodynamic problems have a Toeplitz structure that leads to a considerable speed up during the execution, especially with parallel computation. From the point of view of the calculation time, this makes it easier to manage the increase of linear system degrees of freedom due to refinements of the space-time discretization steps.

\section{ACKNOWLEDGEMENT}

This work has been supported by INdAM, Italy, through granted GNCS research projects.

\section{REFERENCES}

[1] Bécache, E. \& Ha Duong, T., A space-time variational formulation for the boundary integral equation in a $2 \mathrm{~d}$ elastic crack problem. ESAIM: Mathematical Modelling and Numerical Analysis, 28(2), pp. 141-176, 1994.

[2] Frangi, A. \& Novati, G., On the numerical stability of time-domain elastodynamic analyses by BEM. Computer Methods in Applied Mechanics and Engineering, 173(34), pp. 403-417, 1999.

[3] Aimi, A., Desiderio, L., Diligenti, M. \& Guardasoni, C., Application of energetic BEM to $2 \mathrm{D}$ elastodynamic soft scattering problems. Communications in Applied and Industrial Mathematics, 10(1), pp. 182-198, 2019.

[4] Andersen, L., Linear Elastodynamic Analysis, Lecture Notes, Department of Civil Engineering, Aalborg University, 2006.

[5] Aimi, A., Diligenti, M., Guardasoni, C., Mazzieri I. \& Panizzi, S., An energy approach to space-time Galerkin BEM for wave propagation problems. International Journal for Numerical Methods in Engineering, 80(9), pp. 1196-1240, 2009. 
[6] Aimi, A., Di Credico, G., Diligenti, M. \& Guardasoni, C., Highly accurate quadrature schemes for singular integrals in energetic BEM applied to elastodynamics. Journal of Computational and Applied Mathematics (submitted).

[7] Monegato, G. \& Scuderi, L., Numerical integration of functions with boundary singularities. Journal of Computational and Applied Mathematics, 112(1-2), pp. 201214, 1999.

[8] Aimi A., Diligenti M., Monegato G., New numerical integration schemes for applications of Galerkin BEM to 2-D problems. International Journal for Numerical Methods in Engineering, 40(11), pp. 1977-1999, 1997. 